\title{
Morel-Lavallée lesion of the thigh: characteristic findings on US
}

\author{
Arabinda K. Choudhary • Sosamma Methratta
}

Received: 10 November 2009/Revised: 14 January 2010 /Accepted: 5 February 2010/Published online: 9 March 2010

(C) Springer-Verlag 2010

A 12-year-old boy presented for evaluation 3 weeks after an ATV accident. Although no traumatic injury was initially noted, he developed progressive left thigh swelling and blisters 2 weeks after the injury. US identified a collection in the subcutaneous tissues with hyperechoic material (arrowheads) along the wall (Fig. 1). Serous collections were drained at surgery; cultures were negative. Because of continued drainage of lymphatic-appearing fluid, the child was treated with Sotradechol foam injection and doxycycline twice, with improvement.

The Morel-Lavallée lesion is an internal degloving type of injury with separation of the hypodermis from the underlying deep fascia. This results in disruption of the vascular and lymphatic plexus and a cavity is created, filling with blood, lymph, liquefied fat and necrotic debris [1]. US demonstrates a collection in the subcutaneous region with hyperechoic nodules corresponding to remnants of fat. If there is history of shear injury to the soft tissue with chronic recurrent fluid collection and imaging features of subcutaneous collection with fat lobules along the wall, the diagnosis of Morel-Lavallée lesion should be suspected.

A. K. Choudhary $(\varangle) \cdot$ S. Methratta

Department of Radiology, Penn State College of Medicine,

The Milton S. Hershey Medical Center,

500 University Drive, Box 850, Hershey, PA 17033-0850, USA

e-mail: achoudhary@hmc.psu.edu

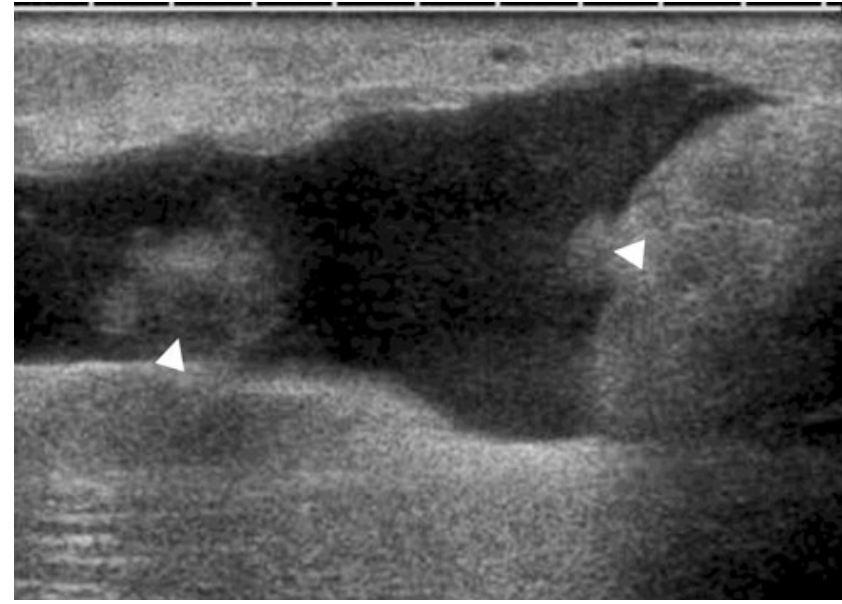

Fig. 1 Longitudinal US image through the left thigh

Treatment options include serial aspiration and, if unsuccessful, sclerodesis. Early identification is important, as delay can increase incidence of infection-related morbidity.

\section{Reference}

1. Puig J, Pelaez I, Baños J et al (2006) Long-standing Morel-Lavallée lesion in the proximal thigh: ultrasound and MR findings with surgical and histopathological correlation. Australas Radiol 50:594-597 